\title{
COVID-19 impact on early career investigators: a call for action
}

Ross L. Levine $\mathbb{1}^{1 凶}$ and W. Kimryn Rathmell ${ }^{2 凶}$

The COVID-19 pandemic has broadly impacted biomedical research and health care. Here we discuss current challenges for the cancer research community as they apply to early career investigators (ECls). We propose a series of collaborative initiatives aimed to sustain $\mathrm{ECls}$ and preserve and accelerate the ability to innovate with long-lasting impact.

The current COVID-19 pandemic has had a pervasive effect, including an unprecedented toll on health, the economy and education worldwide. The impact of this crisis on medical care delivery, disrupting the health-care economy in unprecedented ways, and causing a near-complete halt to basic and translational biomedical research is substantive. While the damage is widespread, early career investigators (ECIs) are at highest risk to experience a negative impact. However, this crisis also reveals opportunities to innovate in creating solutions that accelerate research programmes in the short and long term.

From our perspective, the pause in laboratory and clinical research in the US owing to COVID-19 has lasted more than 2 months and is only resuming under careful, orchestrated guidelines with an uncertain future. This disruption to research presents itself in varied ways in regions across the world. Across the cancer science space, this interruption has been creating uncertainty and suspended states of animation that are particularly detrimental to young investigators. ECIs, including $\mathrm{PhD}$ students, postdoctoral researchers, clinical fellows and junior group leaders, are in a time of steep acceleration with respect to training milestones, protected time, fellowship funding and promotion. Reduced productivity during this time can produce an undesirable 'flattening of the curve' that is potentially unrecoverable.

It is heartening to see ECIs prioritizing data analyses, grant and/or manuscript preparation and reviewing journals during their time out of the lab as an effort to maintain momentum, as they balance their career responsibilities with a daunting set of demands related to increased personal responsibilities during the pandemic $^{1}$. Family care and/or sustaining community ties may also be disproportionately carried by ECIs, particularly impacting women, and the set of challenges faced by investigators who are from minority groups may be amplified. Moreover, clinical trainees, with in-demand skillsets, are necessarily being pulled from protected research time to provide clinical care to patients with
COVID-19. These challenges further threaten the careers and trajectories of the next generation of cancer investigators.

It is also worrisome that job opportunities for ECIs are rapidly diminishing during the pandemic. Academic institutions are facing unprecedented financial challenges with reduction in clinical revenue and philanthropic support. Efforts to counteract these challenges, for example hiring freezes, slowdowns in hiring and/or salary reductions may persist for years. Moreover, the international nature of the COVID-19 pandemic is adding barriers for those who require a visa and/or international travel to continue their careers. The resulting 'back-up' will cause ECIs to stay longer in current positions than intended or end up in limbo between assignments. ECIs may prematurely depart to pursue opportunities in industry or other arenas. The current landscape has the potential to result in irreparable harm to a generation of talented scientists and derail the impressive progress we have seen in cancer research in the past decade.

The funding landscape for cancer research in the US context, which is likely applicable to other contexts, also presents daunting challenges. Universities and academic research and/or clinical institutions will be operating with reduced resources to provide essential institutional support for hiring, internal grants and new programmatic initiatives in the context of reduced clinical and endowment revenues. Moreover, non-profit organizations that provide foundation grants for research, which ECIs often depend on, are seeing a precipitous reduction in donations. This has resulted in reductions in ongoing grant support and a decline in funding opportunities, which may continue for an extended duration. Therefore, we foresee a major gap in support for salary, protected time and pivotal investigations needed for the next generation to develop robust and durable research programmes.

Lastly, we regret the unique impact of social distancing that this pandemic is having on mentoring and networking opportunities for ECIs. Scientific meetings are 
often the venue for ECIs to present work, meet and network with current and future leaders in their field and learn about cutting edge science from across the world. This impact is particularly felt by ECIs at institutions without robust scientific networks or career development programmes, such that it can disproportionately impact those with less institutional support and a less vibrant local research environment.

What can we do in light of these headwinds? We see this as an existential moment to galvanize the international cancer research community to collective action. We believe every person can contribute and propose that collective efforts focus on these six core areas.

\section{Increase connectivity in our field}

There has been a heartening move to increased dissemination of scientific knowledge during the pandemic, including online lectures, increased use of preprints and online discussions of cancer science and career development issues. We need to see further extension of these opportunities to break down silos and ensure that young investigators have a chance to present their science and interact with international leaders in our field in silico.

\section{Enhance opportunities in meetings}

Whether in person or virtual, scientific meetings have always been a tenuous balance between presentations by established leaders in the field and opportunities for ECIs to make their mark. We need to acutely and permanently change this balance and increase opportunities for young investigators to give invited and selected talks at meetings with a commensurate reduction in presentations by senior researchers. This will result in increased diversity and exposure for investigators looking to secure their next training position, their first grant or promotion.

\section{Broaden mentoring opportunities}

Local mentoring is critical and not easily replaced, and many ECIs are suffering owing to reduced interaction with local mentors and no exposure to mentors at outside institutions. We need to develop innovative approaches to facilitate broader mentoring opportunities. For example, one of us (R. L. L.) has invited young investigators to join for virtual one-on-one career and/or science discussions or joint lab meetings. In addition, organizations such as the US Department of Defense Kidney Cancer Research Program have initiated national academy style mentorship programmes to support early career networks and distance mentoring ${ }^{2}$. Such programmes are nascent, and we must seek opportunities to extend mentoring and scientific networks, with feed-forward benefits to the entire community.

\section{Increase support and flexibility thereof}

Most foundations and government funders have shown remarkable flexibility given the pause in research, but we cannot forget that salary and core research expenses (for example, animal care) continue without commensurate productivity. As funding allows, we propose that career awards be extended in duration and in the period of support to allow investigators to recover scientific progress and achieve career development goals.

\section{Transparency in the hiring process}

One of the hardest things about the academic hiring process is the relative opacity, with little information on the priorities, positions or resource availability for prospective applicants. We need 'radical transparency' in the hiring process, such that ECIs can obtain a clearer sense of the number and type of opportunities available at each career stage with clear points of contact for open positions. Moreover, institutions need to enable flexibility for ECIs who cannot transition to their next position owing to hiring freezes or visa restrictions, or we will lose a generation of our best and brightest.

\section{Using our collective voice}

On a broader scale, we are at a critical moment where the public has never been as cognizant of science and medicine as they are during the pandemic. We must seize this moment to engage lay people in our respective communities on the importance of science and medicine and the need for increased, longer-term investment into medical research to benefit all of society, to engage future generations to pursue careers in cancer research and to encourage active participation of people affected by cancer in research and clinical trials.

We believe bold innovations are essential to not only preserve careers of the next generation of cancer innovators, but to have long-lasting impact that allows cancer science to emerge from this devastating crisis stronger, more inclusive and forward thinking to make even greater strides in cancer.

1. Minello, A. The pandemic and the female academic. Nature https://doi.org/10.1038/d41586-020-01135-9 (2020).

2. Choueiri, T. K. et al. Summary from the first Kidney Cancer Research Summit, September 12-13, 2019: a focus on translational research. J. Natl Cancer Inst. https://doi.org/10.1093/jnci/djaa064 (2020).

Acknowledgements

We thank members of the Levine Lab (Bobby Bowman, Aaron Viny, Linde Miles, Sheng Cai and Andrew Dunbar) for their perspective as trainees/ colleagues and Ushma Neill for her comments and guidance. R.L.L. is supported in part by Memorial Sloan Kettering Cancer Center Support Grant/ Core Grant P30 CA008748. W.K.R. is supported by NIH grants K12CA090625 and T32CA217834.

\section{Competing interests}

R.L.L. is on the supervisory board of Qiagen and is a scientific advisor to Loxo/Lilly, Zentalis, Imago, Mana Therapeutics, Auron, Ajax, Syndax, C4 Therapeutics and Isoplexis. He has consulted for Incyte, Syndax, Celgene and Roche. He receives research support from Prelude Therapeutics and has reviewed grants for Celgene and Gilead. W.K.R. receives research support from Incyte and has clinical trial support from Merck, Genentech, BMS Pfizer, Novartis, Incyte and Calithera. 